\title{
Successful partial cochlear implantation in a patient with relapsing polychondritis
}

\author{
Badi Aldosari ${ }^{1,2}$ \\ 1 Department of ORL \& Head and Neck surgery, College of Medicine, King Saud University, Riyadh, Saudi Arabia \\ 2 Department of ORL \& Head and Neck surgery, CHU Nord, Marseille, France \\ Badi Aldosari, ORCID: 0000-0001-9865-3795
}

\begin{abstract}
The author reports on a case of relapsing polychondritis in which partial insertion of a cochlear implant has been successful. A woman with known relapsing polychondritis gave a history of increasingly severe auditory loss of sensorineural type occurring bilaterally. Previous repeated treatments with corticosteroids and immunosuppressive medications had been attempted. A right-sided cochlear implantation with partial electrode array insertion was carried out due to the patient's cochlea being obliterated. The outcome was excellent and the improvement in
\end{abstract}

hearing was maintained. Relapsing polychondritis (RP) is a rarely-seen disorder, the aetiology of which is obscure, but which results in inflammation within cartilage throughout the entire body. The cochlea may become obliterated by this process. Cochlear implantation may be needed in such circumstances. The successful outcome in this case shows a potential role for cochlear implantation in individuals with inner ear disorders produced by immune disorders or relapsing polychondritis.

Keywords: Polychondritis, relapsing, sensorineural hearing loss, cochlear implantation.
Jaksch-Wartenhorst may have been the first person to describe the condition currently termed relapsing polychondritis (RP), writing in $1923 .^{[1]} \mathrm{RP}$ is a rare condition which features recurrent exacerbations of inflammation affecting cartilage. The pathogenic basis and aetiology of RP is uncertain. Some $40-50 \%$ of individuals with RP develop conditions affecting the inner ear at some point in the disorder. ${ }^{[2,3]}$ This report is of a case of RP in which severe auditory loss had occurred, affecting both ears, and of sensorineural type. Previous treatment with corticosteroids and immunosuppressive medications had been to no avail. A cochlear device was implanted successfully, with partial electrode placement.

\section{Case report}

A woman aged 53 years was referred to an ENT clinic in 2004 with a history of profound auditory loss of sensorineural type occurring bilaterally, secondary to RP. She also had vertigo. Total deafness had been present in the left ear for 15 years, and in the right for two months. A diagnosis of RP was reached due to chondritis of the nose, a non-erosive polyarthropathy with negative serology, abnormal vestibulo-cochlear function and appropriate histopathological appearances. Pure-tone audiometric analysis indicated bilateral total deafness. Neither corticosteroid nor immunosuppressant treatment had restored hearing. As expected,
Correspondence: Badi Aldosari

Department of ORL \& Head and Neck surgery, College of Medicine, King Saud University, Riyadh, 11461, Saudi Arabia. E-mail: badi.aldosari@gmail.com
Online available at: www.entupdatesjournal.or

Received: 28.06.2019; Accepted: 25.07.2019 
there was no response on either auditory brain stem response or distortion product otoacoustic emission testing. The temporal bone had been imaged using computed tomography (CT) in 2004, as shown in Figure 1. The appearances were interpreted as indicating an intact cochlea on the right, whilst also showing that the left cochlea was partially obstructed.

It was proposed that a cochlear device be implanted in the right ear. An aortic aneurysm required surgery before the device could be implanted, entailing a delay until 2006. The temporal bone was re-scanned (Figure 2), the appearances at that point showing partial obstruction of the basal turn of the cochlea on the right side, and a more significant obstruction on the left. Right-sided cochlear implantation was performed in 2006 using a Digisonic SP cochlear implant from the Neurelec Company. As we found an obliterated basal turn in the cochlea, the device was partially introduced starting at the second turn of the cochlea. Seven electrodes out of 20 were introduced, and their placement confirmed by transorbital-ocular view.

One month later, the Digisonic SP processor was fitted. Six electrodes were found to be active. Pure tone audiometry showed good thresholds of around $20 \mathrm{~dB}$ between frequencies of $250-4000 \mathrm{~Hz}$, with the cochlear implant operating in a free field. Intelligibility was around $30 \%$ with the cochlear implant alone. The patient reported a high level of satisfaction with the device, which she was using throughout the day. In 2011, the patient benefited from an upgrade to a Saphyr SP processor from the Neurelec Company. There was a definite improvement in the result obtained. Intelligibility with Lafon lists of monosyllabic words was around $50 \%$ at $65 \mathrm{~dB}$ with the cochlear implant alone, and reached $94 \%$ with the cochlear implant used in conjunction with lip reading. The score on the MMBA battery sentences was $41 \%$ at $65 \mathrm{~dB}$ with the cochlear implant alone, and $90 \%$ with the cochlear implant used in conjunction with lip reading. The patient scored 205 on the Nijmegen cochlear implant questionnaire.

\section{Discussion}

$\mathrm{RP}$ is a disease affecting multiple body systems and has an association with autoimmune disorders such as Sjögren's syndrome, systemic lupus erythematosus and rheumatoid arthritis. The condition was first recognized by Jaksch-Wartenhorst in 1923, being described as a polychondropathy. ${ }^{[1]} \mathrm{RP}$ presents with chondritis affecting the pinna, joint inflammation, symptoms affecting the larynx

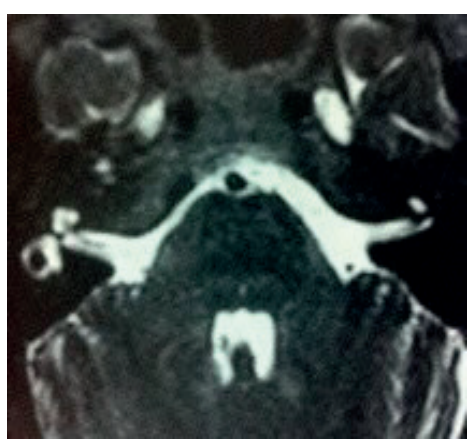

Figure 1. The CT image shows a normal cochlea on the right side and a partial obstruction on the left side.

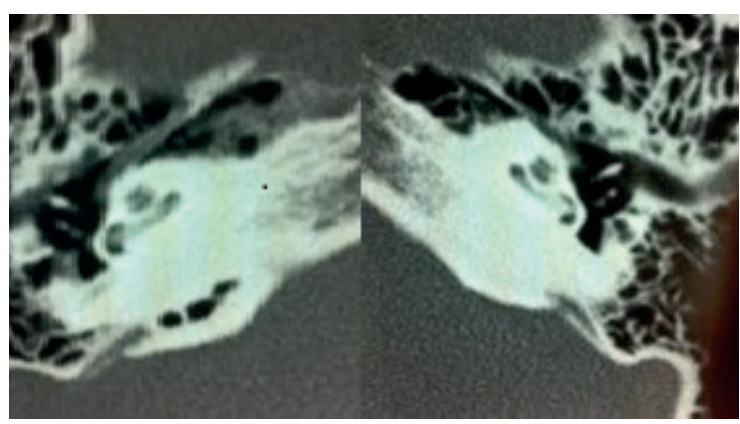

Figure 2. The CT image shows a partial obstruction of the basal turn of cochlea.

and trachea, chondritis of the nose, eye inflammation, symptoms arising from the audiovestibular apparatus and cardiovascular problems. ${ }^{[4]}$ The rate of inner ear complications in RP sufferers is thought to be $40-50 \%$. Auditory loss of sensorineural type may affect one or both sides and the loss may worsen slowly or very rapidly, with resulting cophosis. ${ }^{[5,6]}$ Exactly how the inner ear complications unfold is not currently known. Given that the inner ear does not contain cartilaginous tissue, it is theorised that a different mechanism must be responsible, such as vasculitis of the internal auditory artery, affecting the cochlear or vestibular branches. ${ }^{[2]}$ Hoshino et al ${ }^{[7]}$ examined temporal bone taken from a case of RP in which deafness had rapidly occurred in both ears. They suggest that viral labyrinthitis occurring alongside RP led to cophosis, as the histological appearances showed encapsulation of the tectorial membrane, and severe degeneration of the organ of Corti, as well as of sensory neurons. Miyazawa et al ${ }^{[8]}$ used immunohistochemical techniques to highlight type 2 collagen in 
histological sections obtained from three cases of RP in which RP had led to sensorineural type auditory loss. They discovered that degeneration particularly affected type 2 collagen in the tectorial membrane, where it is abundant, and thus, they suggest, degeneration can be linked to auditory dysfunction. Type 2 collagen is found in various structures of the external and middle ear: the pinna, the cartilage supporting the external auditory meatus, the ear drum and the anulus of the ear drum. ${ }^{[9]}$

Issinget et al ${ }^{[10]}$ performed serology for anti-labyrinthine antibodies, finding these were positive in a case of $\mathrm{RP}$ where there was impairment of auditory and vestibular function. Circulating immunoglobulins or T lymphocytes targeting the self may produce an inflammatory response and apoptosis in the inner ear. Schuhknecht ${ }^{[1]}$ observed that the inner ear lacks cartilaginous structures and suggested that a destructive vasculitis of the labyrinthine artery, or branches thereof, was the pathological mechanism. Such an explanation fits with the observation that systemic necrotizing vasculitis can occur in RP. ${ }^{[12]}$ Whilst auditory loss of sensorineural type does affect $40-50 \%$ of RP sufferers, the majority of such cases do not develop severe auditory impairment. ${ }^{[13]}$ Usually, the therapeutic interventions used for RP, i.e. nonsteroidal anti-inflammatory drugs, corticosteroid and immunosuppressive agent administration, do partially rectify or protect auditory function in RP cases. ${ }^{[14]}$ But rare cases also occur in which auditory loss secondary to RP is not responsive to the usual therapeutic measures. Implantation of a cochlear device has been found beneficial previously in cases of auto-immune ear disorders, namely systemic lupus erythematosus, Cogan's syndrome, Buerger's disease and related disorders. ${ }^{[15]}$ Cochlear implants work by stimulating the para-cochleal spiral ganglion directly. They are potentially highly effective in cases where cochlear function is impaired. One such scenario would be RP resulting in cophosis. Nevertheless, as may happen when there is ossification of the cochlea for other reasons, such as meningitis, cochlear obliteration may render the implantation of a cochlear device highly challenging. Thus, a high level of clinical vigilance is needed in any case where cochlear obliteration is deemed a risk, so that the device may be implanted prior to the point where cochlear obliteration prevents the siting of electrodes. In the case reported here, good results were still obtainable, despite only partial implementation (five electrodes out of 20 were possible). This outcome is highly encouraging, as the results are superior to other possible options, including an auditory brainstem implant. In clinical practice, whenever possible a cochlear implantation is preferable, even if only partial, to an auditory brainstem implant.

\section{Conclusion}

Relapsing polychondritis is a rarely seen disorder, the ae-tiology of which is obscure, but which results in inflam-mation within cartilage throughout the entire body. The cochlea may be obliterated by this pathological process. Cochlear implantation may be needed in such circum-stances. The successful outcome in this case shows a poten-tial role for cochlear implantation in individuals with inner ear disorders produced by immune disorders or relapsing polychondritis.

Informed Consent: Written informed consent was obtained from the patient who participated in this study.

Author Contributions: Designing the study - B.A.; Collecting the data - B.A.; Analyzing the data - B.A.; Writing the manuscript - B.A.; Confirming the accuracy of the data and the analyses - B.A.

Conflict of Interest: The author has no conflicts of inter-est to declare.

Financial Disclosure: The author declares that this study has received no financial support. 


\section{References}

1. Jaksch-Wartenhorst R. Polychondropathia. Wien Arch Inn Med 1923;6:93-100.

2. McAdam LP, O'Hanlan MA, Bluestone R, Pearson CM. Relapsing polychondritis: prospective study of 23 patients and a review of the literature. Medicine (Baltimore) 1976;55:193-215

3. Zeuner M, Straub RH, Rauh G, Albert ED, Schölmerich J, Lang B. Relapsing polychondritis: clinical and immunogenetic analysis of 62 patients. J Rheumatol 1997;24:96-101.

4. Pearson CM, Kline HM, Newcomer VD. Relapsing polychondritis. N Eng J Med 1960;263:51-8.

5. Trentham DE, Le CH. Relapsing polychondritis. Ann Intern Med 1998;129:114-22.

6. Cody DT, Sones DA. Relapsing polychondritis: audiovestibular manifestations. Laryngoscope 1971;81:1208-22.

7. Hoshino T, Kato I, Kodama A, Suzuki H. sudden deafness in relapsing polychondritis: a scanning electron microscopy study. Acta Otolaryngol 1978;86:418-27.

8. Miyazawa T, Ino Y, Murakami Y, Ishii T. Immunohistochemical study on the temporal bones from patients with relapsing polychondritis. Jibimeneki Arerugi 2000;18:66-7.
9. Tomoda K, Yamashita T, Kumazawa T, Yoo TJ. Type II collagen distribution in the middle and inner ear: immunohistochemical studies. Ear Res Jpn 1984;15:199-202.

10. Issing WJ, Selover D, Schulz P. Anti-labyrinthine antibodies in a patient with relapsing polychondritis. Eur Arch Otorhinolaryngol 1999;256:1636.

11. Schuknecht H. Ear pathology in autoimmune disease. Adv Otorhinolaryngol 1991;46:50-70.

12. Michet C. Vasculitis and relapsing polychondritis. Rheum Dis Clin North Am 1990;16:441-4.

13. Bachor E, Blevins NH, Karmody C, Kuhnel T. Otologic manifestations of relapsing polychondritis. Review of literature and report of nine cases. Auris Nasus Larynx 2006;33:135-41.

14. McCaffrey TV, McDonald TJ, McCaffrey LA. Head and neck manifestations of relapsing polychondritis: review of 29 cases. Otolaryngology 1978;86:473-8.

15. Quaranta N, Bartoli R, Giagnotti F, Di Cuonzo F, Quaranta A. Cochlear implants in systemic autoimmune vasculitis syndromes. Acta Otolaryngol Suppl 2002;548:44-8. 